\title{
Temperature Field Online Reconstruction for In-Service Concrete Arch Dam Based on Limited Temperature Observation Data Using AdaBoost-ANN Algorithm
}

\author{
Zhuoyan Chen $\mathbb{D}^{1,2}$ Dongjian Zheng $\mathbb{D}^{1,2}$ Jiqiong $\mathrm{Li}^{3}{ }^{3} \mathrm{Xin}_{\mathrm{Wu}}{ }^{1,2}$ and Jianchun Qiu ${ }^{4}$ \\ ${ }^{1}$ State Key Laboratory of Hydrology-Water Resources and Hydraulic Engineering, Hohai University, Nanjing 210098, China \\ ${ }^{2}$ National Engineering Research Center of Water Resources Efficient Utilization and Engineering Safety, Hohai University, \\ Nanjing 210098, China \\ ${ }^{3}$ China Design Group Co., Ltd., Nanjing 210018, China \\ ${ }^{4}$ College of Hydraulic Science and Engineering, Yangzhou University, Yangzhou 225009, China
}

Correspondence should be addressed to Dongjian Zheng; zhengdj@hhu.edu.cn

Received 30 March 2021; Accepted 18 July 2021; Published 23 July 2021

Academic Editor: Nhon Nguyen Thanh

Copyright $\odot 2021$ Zhuoyan Chen et al. This is an open access article distributed under the Creative Commons Attribution License, which permits unrestricted use, distribution, and reproduction in any medium, provided the original work is properly cited.

\begin{abstract}
Temperature is one of the factors affecting the safety operation of concrete arch dams. To accurately reconstruct the temperature field of the concrete arch dam online based on the temperature data of several typical dam sections, this paper proposes the AdaBoost-ANN algorithm. The algorithm uses artificial neural network (ANN) to establish a training set of the measured temperature data and the temperature field of the concrete arch dam obtained by the three-dimensional finite element model; these trained artificial neural networks are used as weak classifiers of the AdaBoost algorithm. Then, the AdaBoost-ANN algorithm is used to establish the mapping relationship between the measured temperature data and the temperature field, and the online reconstruction of the temperature field of the concrete arch dam is realized. The case study shows that the temperature field of the concrete arch dam can be accurately established by AdaBoost-ANN algorithm based on limited temperature observation data. The algorithm is more time-saving and labor-saving than the finite element method and is convenient for online reconstruction of the temperature field and assessment of the safety status of the concrete arch dam.
\end{abstract}

\section{Introduction}

Thermal loads play a vital role in the safety operation of concrete arch dams. By accurately establishing the temperature field of the arch dam and combining the water level and other data, the safety index of the arch dam can be obtained, which is helpful for the safety operation of the arch dam. Temperature not only affects the stress field and the stability of the arch dam, but also affects the deformation field of the arch dam [1-4]. Temperature variations will cause the stiffness, strength, and durability of the concrete dam to decrease. And the temperature field of the concrete arch dam is affected by seasonal temperature variations; temperature changes between summer and winter can sometimes be as high as $75^{\circ} \mathrm{C}$. For example, temperature loads play an important role in the generation and development of cracks in the downstream surface of Daniel Johnson arch dam [5]. The deformation of Schlegeis arch dam and Alto Lindoso arch dam and the cracks on the downstream side of Karaj arch dam are closely related to the change of the instantaneous temperature fields on the downstream surface [6-9]. The transient temperature field change of the arch dam caused by daily temperature change has a significant impact on the daily deformation amplitude of the arch dam $[10,11]$. Therefore, it is necessary to study an online arch dam temperature field reconstruction method.

At present, there are two main methods to reconstruct the temperature field of the in-service arch dam. One is using the actual temperature values measured by the thermometers embedded in dam concrete to extrapolate the 
temperature field of the dam body directly [12]. It is stated in the design specification for concrete arch dams that the above method is feasible when the temperature data is insufficient [13]. Li [14] established the concrete dam temperature field by using the temperature values inside the dam to extrapolate the temperature values of the dam body. However, generally only a few typical sections are equipped with thermometers, and the distance between these dam sections usually reaches tens of meters. Therefore, the measured values of the thermometers could not accurately reflect the temperature field of the dam sections which have no thermometers and far from the measuring points. There is a large error between the temperature field of the dam reconstructed by the temperature extrapolation method and the actual temperature field of the dam.

The other is using the numerical simulation method to establish the temperature field of the in-service concrete arch dam [15-20]. The temperature field of the concrete arch dam changes with the surrounding environment due to the actions of convection, radiation, and evaporative cooling. These factors need to be considered in the numerical simulation of the temperature field of a concrete arch dam $[8,21]$. Furthermore, continuous simulation of the working performance of the dam from the construction stage to the service stage is required, especially for the super-high arch dams that are usually poured layer by layer [22-25]. Therefore, the commonly used in this method is using the finite element method (FEM) to determine the parameters of the model based on the measured data and then combining with the boundary conditions observed to reconstruct the temperature field. Sheibany and Ghaemian [7] developed a three-dimensional finite element model to study the temperature and thermal stress distribution of the concrete arch dam during operation, and the effects of environmental action on the thermal stress analysis of Karaj concrete arch dam were concluded. Zvanut et al. [26] conducted the heat transfer analysis of Moste concrete gravity-arch dam, located in northwest Slovenia, after a new sophisticated monitoring system had been established. The results show that the measured temperature values of the dam are very consistent with the temperature values obtained by the numerical simulation method. However, using the numerical simulation method mentioned above, the temperature field reconstruction of the concrete arch dam is complex and time consuming. Each time the numerical simulation method is used, the boundary conditions and model parameters need to be updated based on the observation data.

To synthesize the advantages of the above methods and mitigate their disadvantages, accurately and conveniently reconstruct the temperature field of the arch dam, and then assess the safety status of the arch dam; this paper intends to use the AdaBoost-ANN algorithm combined with the measured temperature data obtained by thermometers embedded in dam concrete and temperature fields obtained by finite element method based on the measured temperature data during the observation period to reconstruct the temperature field of the in-service concrete dam. The mapping relationship between the temperature fields and the temperature values of measuring points in the arch dam can be established and updated. And the temperature field of inservice concrete arch dam can be quickly reconstructed online based on the limited thermometer measured data, which lays a foundation for determining the safety status of the arch dam in time. To verify the accuracy and superiority of the temperature field model established by the AdaBoostANN algorithm, this paper selects Xiaowan concrete doublecurved arch dam as an example. The results obtained by the AdaBoost-ANN algorithm are compared with the temperature fields extrapolated by the temperature values measured by thermometers inside the dam and the temperature fields obtained by the finite element method, which proves the accuracy and superiority of using the AdaBoost-ANN algorithm to establish the temperature field of the arch dam.

\section{Numerical Simulation Calculation of Temperature Field of Concrete Arch Dams}

2.1. Principle of Temperature Field Calculation and Determination of Boundary Condition. It is known from the theory of heat conduction [27-29] that the three-dimensional unsteady temperature field $T(x, y, z, t)$ should satisfy the following heat conduction differential equation; the corresponding initial conditions and boundary conditions are shown as follows:

$$
\frac{\partial T}{\partial t}=\alpha\left(\frac{\partial^{2} T}{\partial x^{2}}+\frac{\partial^{2} T}{\partial y^{2}}+\frac{\partial^{2} T}{\partial z^{2}}\right)+\frac{\partial \theta}{\partial t}
$$

where $T(x, y, z, t)$ is a temperature field function; $t$ is the time and $(x, y, z)$ is the coordinate of the corresponding point; $\alpha$ is the temperature coefficient of concrete; and $\theta$ is the absolute temperature rise of concrete.

The corresponding initial conditions and boundary conditions are shown as follows:

$$
\begin{aligned}
\left.T\right|_{t=0} & =T_{0}(x, y, z), \\
\Gamma_{1}: T(x, y, z, t) & =T_{b}(x, y, z, t), \\
\Gamma_{2}:-\lambda \frac{\partial T}{\partial n} & =\beta\left(T-T_{a}\right), \\
\Gamma_{3}: \frac{\partial T}{\partial n} & =0,
\end{aligned}
$$

where $T_{0}(x, y, z)$ is an initial temperature field; $\Gamma_{1}$ is a temperature boundary condition; $\Gamma_{2}$ is a surface heat exchange boundary condition; and $\Gamma_{3}$ is an adiabatic boundary condition; $T_{b}(x, y, z, t)$ is the boundary temperature; $T_{a}$ is the ambient temperature; $\lambda$ is the thermal conductivity; and $\beta$ is the surface exotherm coefficient.

For the concrete arch dam at the beginning of operation, the internal hydration heat of the concrete has not been completely dissipated, at this time $(\partial \theta / \partial t) \neq 0$. For the concrete arch dam during long-term operation, the hydration heat has been completely dissipated, at this time $(\partial \theta / \partial t)=0$.

To obtain the unique solution of the above heat transfer equations, the initial temperature distribution of the concrete arch dam must be defined, and the thermal boundary 
conditions of the concrete air, concrete water, and concrete foundation interfaces must also be specified. The heat flux of the dam surface in contact with air is the sum of the energy from convection, long-wave radiation exchange, solar energy, and evaporative cooling [30-32]. And it is usually assumed that the temperature of the dam surface is the same as the temperature of the water in contact with it, and an adiabatic condition is used at the dam foundation interface. Anyways, the thermal boundary conditions are complex problems. This paper intends to use the polynomial function to fit the temperature distribution of the measuring points in the dam and obtain the boundary temperature of the dam. Suppose that at a certain elevation of the arch dam, the distance between the thermometer in the dam and the upstream dam surface of the elevation is $l$, and the measured temperature value is $T_{0}(l)$. Since the temperature of the dam is generally high-order continuous change along the upstream and downstream directions [33], and $T_{0}(l)$ has any order derivative in a certain neighborhood $U\left(l_{0}\right)$ at the point $l_{0}$; then $T_{0}(l)$ at this elevation can be expressed as follows:

$$
T_{0}(l)=a_{0}+a_{1} l+a_{2} l^{2}+\cdots+a_{n} l^{n}+\cdots .
$$

To meet the accuracy requirements, this paper selects the fourth-order polynomial as shown in the following equation to fit the measured temperature value $T_{0}(l)$ :

$$
T_{0}(l)=a_{0}+a_{1} l+a_{2} l^{2}+a_{3} l^{3}+a_{4} l^{4} .
$$

The polynomial regression coefficient in equation (5) is solved to obtain the temperature distribution function at the typical dam section using the measured temperature data, and then the temperature distribution functions obtained at every typical dam section are used to derive the boundary temperature of the corresponding elevation. After obtaining the upper and lower boundary temperature of the typical dam section, the boundary temperature in the dam axis direction and elevation direction can be solved by this method. And the arch dam temperature field can be obtained by using equations (1) and (2).

2.2. Temperature Field Reconstruction by FEM. At the observation time $t_{0}$, the specific calculation steps of the temperature field are as follows: Establish the finite element model of the arch dam temperature field, and set as many unit nodes of the model as possible at the actual temperature measuring points. The temperature field calculation parameters are obtained by inversion of the measured temperature values of the typical dam sections. Considering the hysteresis effect of the ambient temperature on the arch dam, if the observation frequency of the typical section temperature of the arch dam is once per hour (or every day), the time $t_{0}-n$ can be used as the beginning time for calculating the temperature field of the arch dam. The boundary temperature values of the dam surface are calculated by the polynomial regression method mentioned in Section 2.1. The initial temperature field at time $t_{0}-n$ of the arch dam can be approximately obtained from the temperature values of the measuring points by linear interpolation. Then, the initial temperature field at time $t_{0}-n$ and the boundary temperature calculated by equation (5) at time $t_{0}-(n-1)$ can be used to calculate the temperature field at time $t_{0}-(n-1)$ by equation (1) and the finite element model. And thus, the temperature field at time $t_{0}-(n-2), \ldots, t_{0}-1, t_{0}$ can be obtained. In this way, the temperature field of the concrete arch dam can be reconstructed at time $t_{0}$ and the influence of the initial temperature field is reduced after $n$ iterations.

According to the above process, the temperature field of the dam corresponding to the observation time can be obtained, and FEM can be used to accurately establish the temperature field of the arch dam [34]. However, every time the temperature field is established, it is necessary to reanalyze the three-dimensional finite element temperature field and determine the corresponding boundary conditions. This process is very cumbersome and time consuming, so a simpler method to establish the temperature field is needed.

\section{Temperature Field Reconstruction by AdaBoost-ANN}

Assume that the temperature fields of the arch dam at different time periods in service are $\left\{T_{E j}, j=1, \ldots M\right\}$ (M is the number of observations of the dam temperature) obtained by the method mentioned in Section 2.2 and the measured temperature values inside the dam are $\left\{T_{i}, i=1, \ldots N\right\}$ ( $N$ is the number of thermometers embedded inside the dam). The mapping relationship between $\left\{T_{E j}, j=1, \ldots M\right\}$ and $\left\{T_{i}, i=1, \ldots N\right\}$ is as follows:

$$
\left\{T_{E j}\right\} \sim\left\{f_{j}\left(T_{i},(x, y, z)_{i}\right)\right\}, \quad(i=1, \ldots, N ; j=1, \ldots, M),
$$

where $(x, y, z)_{i}$ is the coordinate of the temperature measuring point $i$.

Inputting the measured values of the thermometers into equation (6), the temperature field $\left\{T_{E}(x, y, z)\right\}$ of the concrete dam can be obtained, which simplifies the reconstruction process of the temperature field of the dam.

It can also be seen from equation (6) that the nonlinear mapping relationship between the measured values $\left\{T_{i}, i=1, \ldots N\right\}$ of $N$ thermometers and the temperature fields $\left\{T_{E}(x, y, z)\right\}$ of the dam is complicated. In this paper, the AdaBoost-ANN algorithm which has strong nonlinear mapping ability is used to realize the fast reconstruction of the overall temperature field of the arch dam based on the limited temperature observation data of the concrete arch dam. The principle of the AdaBoost-ANN algorithm is described below.

3.1. Principle of AdaBoost-ANN Algorithm. Artificial neural network (ANN) has advantages in solving nonlinear problems. However, for different parts of the arch dam, the mapping relationship between the temperature observation data and the temperature field is different. When the ANN algorithm is used to map only once, there will be large errors and local overfitting, and the accuracy of the temperature field obtained in this way is usually unacceptable. Therefore, it is necessary to establish a multiple mapping model to 
improve the accuracy. The Adaptive Boosting (AdaBoost) [35] algorithm has advantages in solving multiple mapping problems. By gradual learning, different weights are assigned to weak classifiers with low accuracy, and the weight of each weak classifier is fully considered to obtain a high-precision strong classifier [36, 37]. In view of the characteristics of the above two algorithms, this paper combines the AdaBoost algorithm with the ANN to achieve their respective advantages. And the ANN is used as the weak classifier to reconstruct the temperature field of the arch dam based on limited temperature observation data. In this paper, Backpropagation (BP) neural network which is one of the ANNs is used as weak classifiers.

The BP algorithm is a multilayer feedforward network based on error backpropagation training [38, 39]. The hidden layer has one or more layers of neurons as needed [40]. In this paper, a 3-layer BP network, including the input layer, hidden layer, and output layer, is used to establish the deflection calculation model [41]. The algorithm flow is divided into three steps: network construction, training, and prediction $[42,43]$. The topology of the BP algorithm is shown in Figure 1.

To overcome the slow convergence of the BP algorithm and its tendency to fall into a local minimum [39], this paper introduces a genetic algorithm to optimize the BP algorithm. This is mainly done to establish the fitness function, find the individual factors corresponding to the optimal fitness value through selection, crossover, and mutation operations, and then assign the optimal individual factors to the initial weights and thresholds of the network [44]. The network is trained, and the outputs are given by the prediction function. The temperature observation data and the temperature field results calculated by FEM are substituted into the BP neural network for training, the input layer is the temperature observation data, and the output layer is the temperature field results. Then, the trained BP neural network is substituted into the AdaBoost algorithm.

The weight of the corresponding BP neural network is obtained by training the AdaBoost algorithm. Then, the percentage of the weight value of each BP neural network to the total weight value is taken as the coefficient of this BP neural network (weak classifier). Finally, these weak classifiers are superimposed according to their respective weight values to obtain the final strong classifier.

Before training the AdaBoost-ANN algorithm, it is necessary to determine the error limit $\delta$ between the output value of the $\mathrm{BP}$ neural network and the actual value. When the error between the output value and the actual value is less than the error limit $\delta$, it can be determined that the BP neural network weak classifier classifies the data correctly; otherwise, the classification is incorrect.

3.2. Establishment of AdaBoost-ANN Algorithm. Train multiple BP neural networks and use AdaBoost algorithm to fit these BP neural networks. After $k$ iterations, a strong classifier is obtained. The specific process is as follows.

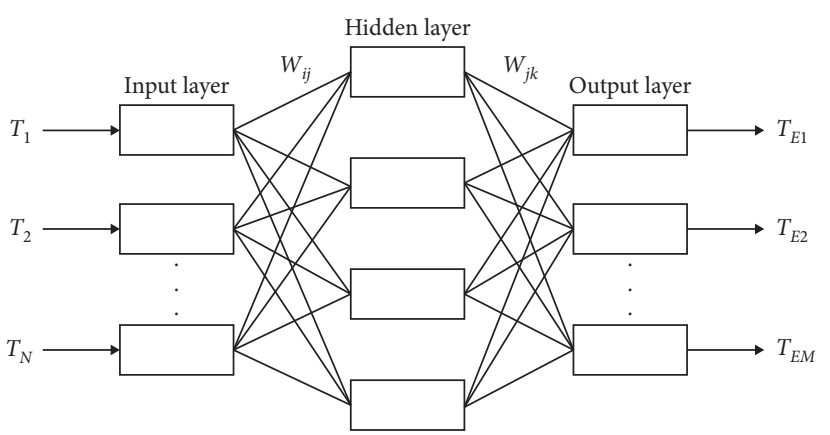

Figure 1: Diagram of the BP neural network topology.

Selecting $m$ sets of data as training samples, and assigning an initial weight to each set of data, the initial weight is

$$
D_{0}(n)=\frac{1}{m}
$$

where $D_{0}(n)$ is the initial weight of the $n$th set of data and $m$ is the total number of samples.

The 3-layer BP neural network model is used as a weak classifier for training. Before training, the number of nodes in the input layer, hidden layer, and output layer of the BP neural network, as well as the transfer function, training function, learning algorithm, and function performance, should be set. And in the $i$ th training, the base classifier $t(i)$ is obtained. Then, compare the error between the actual output and the target output of $t(i)$, and add up the weight $D_{i}(n)$ of the part where the error exceeds $\delta$ to obtain the calculation error $\varepsilon_{i}$. $D_{i}(n)$ is the weight of the $n$th set of data in the $i$ th iteration. It can be seen that $\varepsilon_{i}$ is the sum of weights of the samples misclassified by $t(i)$. The weight $\alpha_{i}$ of the base classifier $t(i)$ can be calculated by the following equation:

$$
\alpha_{i}=\frac{1}{2} \ln \left(\frac{1-\varepsilon_{i}}{\varepsilon_{i}}\right),
$$

And adjust the weight as shown in the following equation:

$$
D_{i+1}(n)=\frac{D_{i}(n)}{Z_{i}} \times \begin{cases}e^{-\alpha_{i}}, & \text { |actual output }- \text { target output } \mid<\delta, \\ e^{\alpha_{i}}, & \text { |actual output }- \text { target output } \mid \geq \delta,\end{cases}
$$

where $Z_{i}$ is calculated as follows:

$$
Z_{i}=\sum_{n=1}^{m} D_{i}(n) \times \begin{cases}e^{-\alpha_{i}}, & \text { |actual output }- \text { target output } \mid<\delta, \\ e^{\alpha_{i}}, & \text { |actual output }- \text { target output } \mid \geq \delta .\end{cases}
$$

Then, repeat the above steps and iterations to obtain $k$ weak classifiers, and accumulate all weak classifiers according to their respective weight values to obtain a strong classifier $T$ as shown in the following equation:

$$
T=\sum_{i=1}^{k} \frac{\alpha_{i}}{\sum_{i=1}^{k} \alpha_{i}} t_{i} .
$$




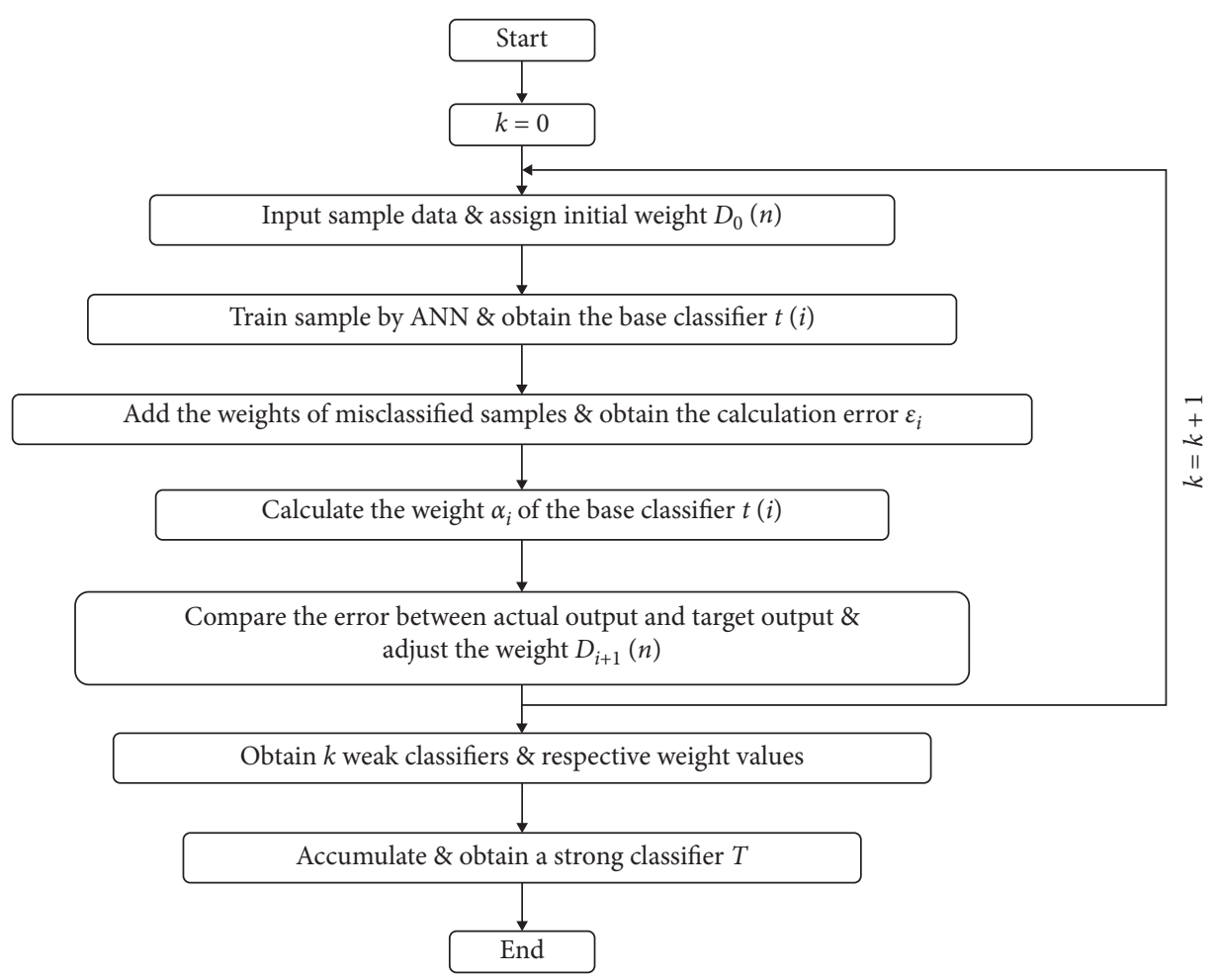

Figure 2: The flowchart of AdaBoost-ANN algorithm.

The flowchart of the AdaBoost-ANN algorithm is shown in Figure 2.

\section{Establishment of Temperature Field of Xiaowan Arch Dam}

To verify the accuracy and superiority of the AdaBoost-ANN algorithm in the establishment of the temperature field of the arch dam, this paper uses the dam temperature extrapolation method, FEM, and the AdaBoost-ANN algorithm to establish the temperature field of Xiaowan Arch Dam. Then, 3 dam temperature field models are compared for verification.

\subsection{Xiaowan Arch Dam Overview. Xiaowan Arch Dam is the} second highest arch dam and the third highest dam in the world. The dam crest elevation is $1245 \mathrm{~m}$, the dam foundation elevation is $950.5 \mathrm{~m}$, the maximum dam height is $294.5 \mathrm{~m}$, the dam crest length is $901.771 \mathrm{~m}$, the top of the crown cantilever width is $12 \mathrm{~m}$, and the bottom of the crown cantilever width is $72.912 \mathrm{~m}$. The arch dam is shown in Figure 3. The reservoir began to impound in November 2007, the first generator was commissioned in September 2009, and the dam was completed in March 2010.

Various instruments have been embedded in Xiaowan arch dam and its foundation to monitor its working status, with 6391 measuring points of various types including thermometers, strain gauges, dummy gauges, joint meter, crack meters, pressure gauges, and multipoint borehole extensometers. A total of 297 thermometers are arranged in $9 \#, 15 \#, 22 \#, 29 \#$, and $35 \#$ dam sections to monitor the temperature of the dam. In addition, many of the above

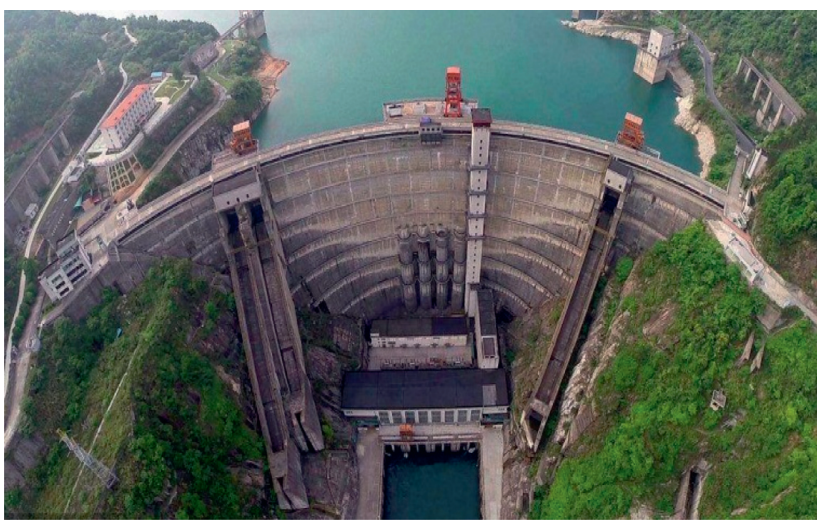

Figure 3: Xiaowan arch dam.

instruments are also equipped with temperature sensors. All instruments are read once a day. Taking the 22\# typical dam section as an example, the thermometer layout is shown in Figure 4.

4.2. Temperature Field Established by FEM. According to the actual situation and geological data, HyperMesh software is used to establish the three-dimensional model of Xiaowan Arch Dam, and Marc software is used to calculate the arch dam temperature field. The boundary conditions of the model are as follows: the upstream length is 1.5 times the height of the dam, the downstream length is 2 times the height of the dam, the extension length of the left and right abutments is 2 times the height of the dam, and the length below the dam foundation is 1.5 times the height of the dam. 


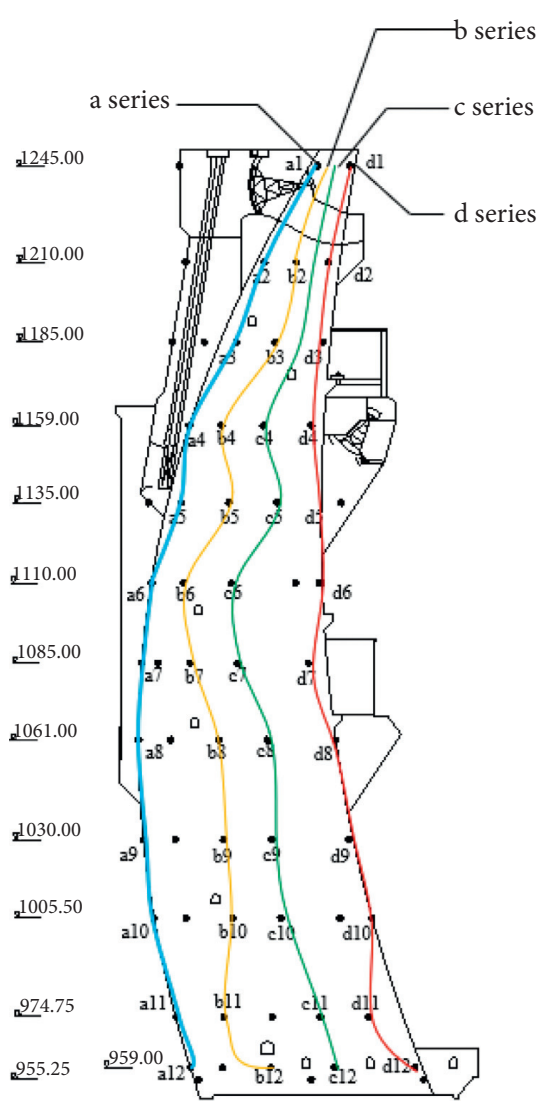

FIgURE 4: Layout of the thermometers in 22\# dam section.

The model mainly simulates the dam shape, structural features, river valley topography, and rock mass structure of the arch dam. The overall finite element model of the arch dam and dam foundation is shown in Figure 5.

To calculate the boundary temperature of the dam, the temperature distribution function of each dam section at different elevations is fitted by the quartic polynomial based on the measured values of the thermometers inside the dam. And the boundary temperature value of the dam body is determined by using the method mentioned in Section 2.1. After the parameters of the finite element model are inversed based on the measured data, the temperature field of the arch dam is reconstructed by the model.

4.3. Temperature Field Established by AdaBoost-ANN Algorithm. AdaBoost-ANN algorithm needs to be trained to establish the temperature field of the arch dam. In this paper, the measured temperature data of 2 years in 2011 and 2012 and the arch dam temperature fields $\left\{T_{E j}, j=1, \ldots M\right\}$ of 5 typical dam sections 9\#, 15\#, 22\#, 29\#, and 35\# obtained from the finite element model are selected as training samples, a total of 7200 sets of data. And the temperature data of the above 5 dam sections in 2013 and the temperature fields obtained from the finite element model are selected to verify the accuracy of the AdaBoost-ANN algorithm.

The input and output data are normalized, and 3-layer BP neural networks are trained by MATLAB software. The input layer is the temperature data $\left\{T_{i}, i=1, \ldots N\right\}$

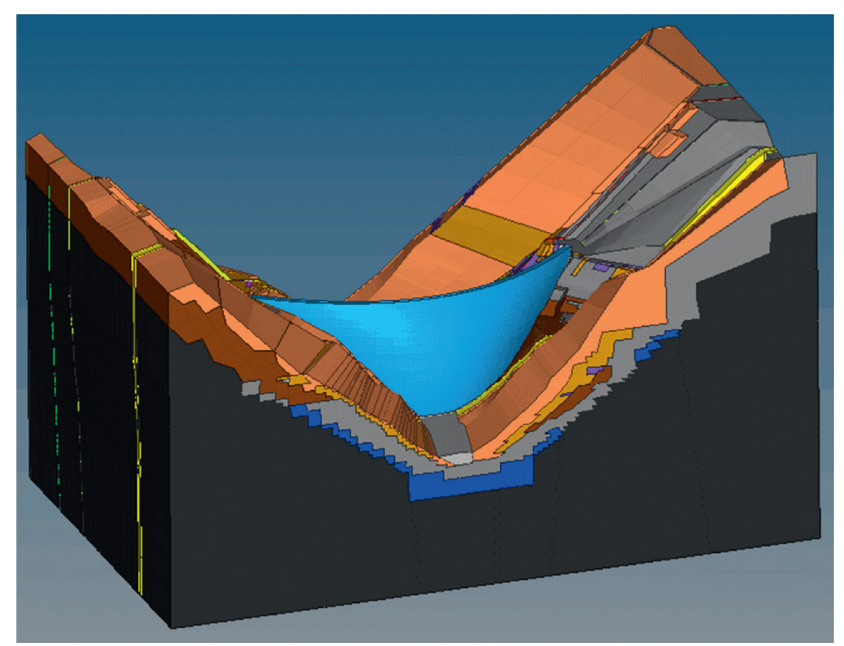

FIGURE 5: Finite element model of the arch dam and dam foundation.

measured inside the dam, the output layer is the temperature field $\left\{T_{E j}, j=1, \ldots M\right\}$ calculated by the finite element model, and the number of hidden layers is determined to be 5 according to Kolmogorov's theorem [44, 45]. The tansig function is selected to be the transfer function of the hidden layer, the sigmoid function is selected as the transfer function of the output layer, and the fitness function of the genetic algorithm is selected as the sum of the absolute values of the prediction errors from the training data.

The number of learning steps is set to 3000 , the learning speed is 0.05 , the momentum factor is 1.05 , and the network global error is set to $10^{-6}$. The parameters of the genetic algorithm were set as follows: a population size of 10 , an evolution frequency of 50, a crossover probability of 0.9 , and a compilation rate of 0.05 . Each weak classifier is given an initial weight, and the sample with a prediction error exceeds 0.1 should be strengthened. The number of iterations of the AdaBoost algorithm is determined by the error variation of the test sample during iteration. In this paper, the error variation of the test sample tends to be stable after the 8th iteration. In order to avoid the phenomenon of overlearning, the number of iterations is set as 8 . After training, this paper obtains $8 \mathrm{BP}$ neural network weak classifiers, and then the AdaBoost algorithm is used to obtain a strong classifier. Finally, the arch dam body temperature can be predicted by the strong classifier, and the temperature field model of the arch dam is established.

\section{Comparison and Analysis of Results}

To verify the effectiveness and superiority of the AdaBoostANN algorithm, the temperature fields of the 5 typical dam sections (9\#, 15\#, 22\#, 29\#, and 35\#) and another 5 dam sections (6\#, 12\#, 18\#, 24\#, and 30\#) obtained from the measured temperature data in 2013 are taken as examples. For the typical elevation of the $22 \#$ dam section, the temperature values calculated by the temperature extrapolation method, FEM, and AdaBoost-ANN are shown in Figure 6. And the temperature field diagrams of the typical sections 


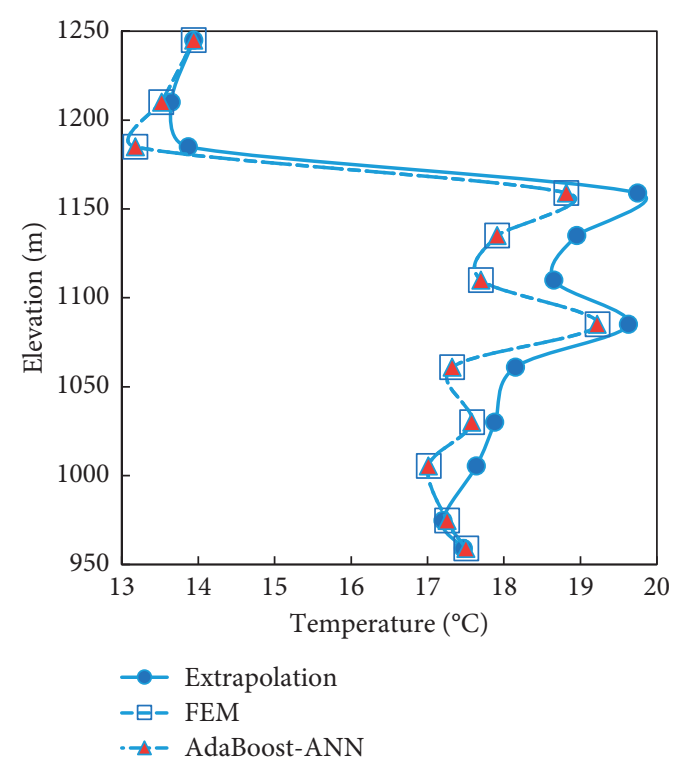

(a)

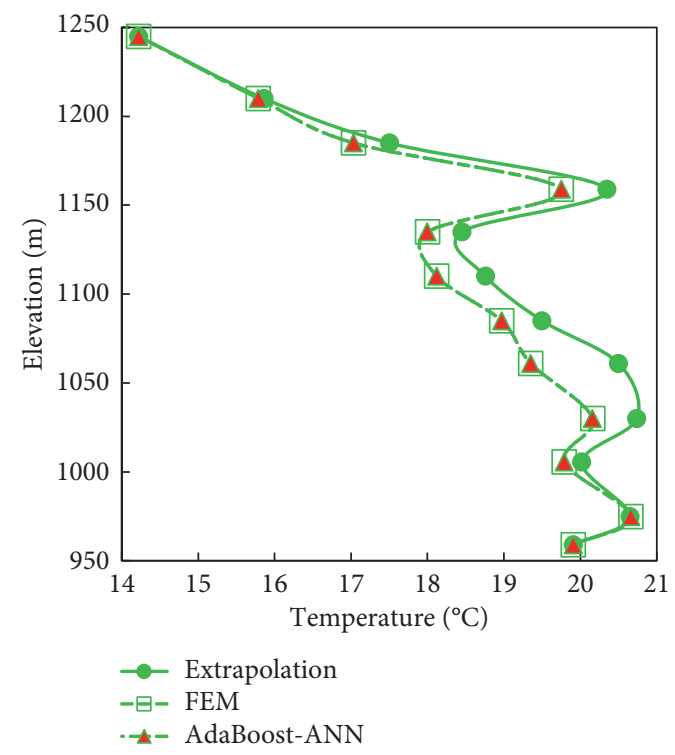

(c)

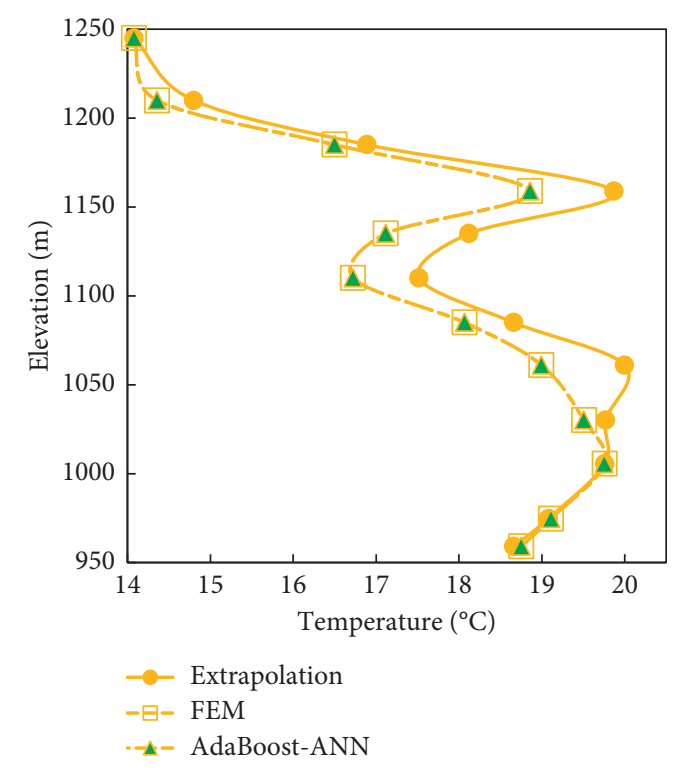

(b)

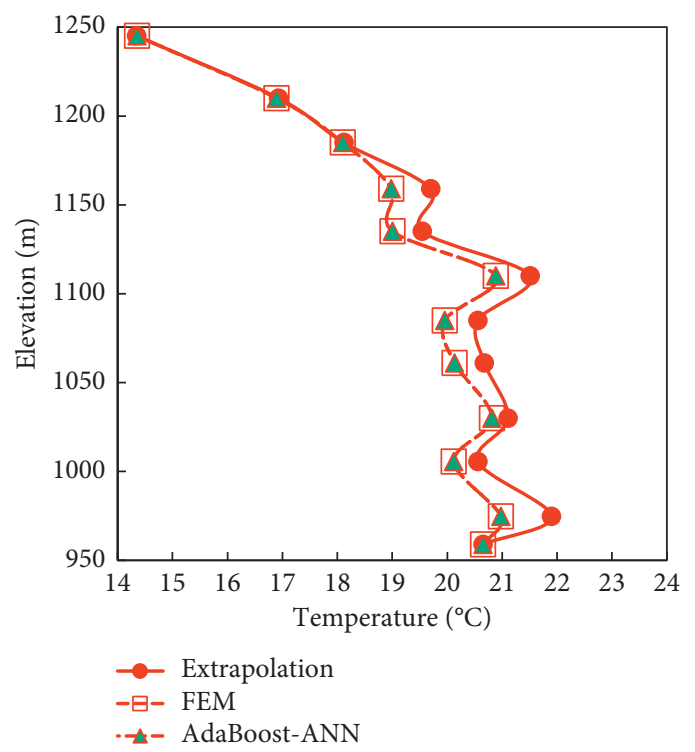

(d)

FIGURE 6: Temperature values obtained by the three methods in this paper. (a) a series temperature values. (b) b series temperature values. (c) c series temperature values. (d) d series temperature values.

calculated by the AdaBoost-ANN algorithm are shown in Figure 7. For the temperature fields of the 5 typical dam sections and another 5 dam sections, the average relative errors of the results obtained by the AdaBoost-ANN algorithm and the temperature extrapolation method relative to the results obtained by FEM are shown in Table 1 .

It can be seen from Figures 6 and 7 that the temperature field of the arch dam can be obtained by the AdaBoost-ANN algorithm and the temperature values obtained by the AdaBoost-ANN algorithm are very close to the results of the FEM. Table 1 shows that for the temperature fields of all dam sections, the relative errors between the results obtained by the AdaBoost-ANN algorithm and the results obtained by the FEM are small, the maximum relative error is less than $1 \%$. The temperature field calculated by the temperature extrapolation method has a large error with respect to the FEM, and the relative errors are almost more than $5 \%$. And the relative errors of the results of the atypical dam sections obtained by the temperature extrapolation method are larger than that of the typical dam sections. This is because the temperature field of the atypical dam section is derived from the relevant data of the typical dam section, which leads to the accumulation of errors and makes the relative errors larger. Under the conditions of the computer used in this paper, the average computational cost of FEM is 50 minutes, and the average computational cost of the AdaBoost-ANN algorithm is 10 minutes. It can be seen that the average computational cost of the AdaBoost-ANN algorithm is only $1 / 5$ of the average computational cost of FEM. 


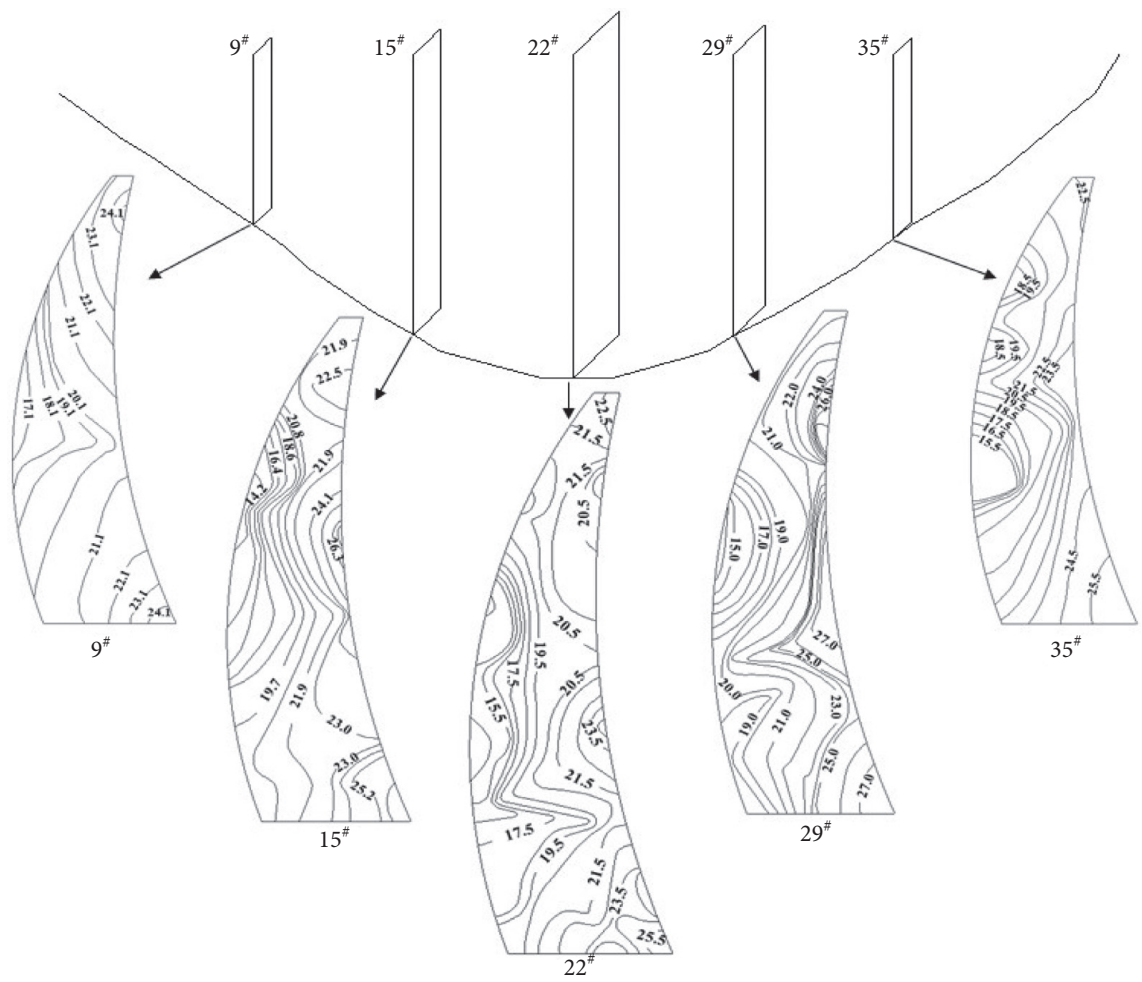

(a)

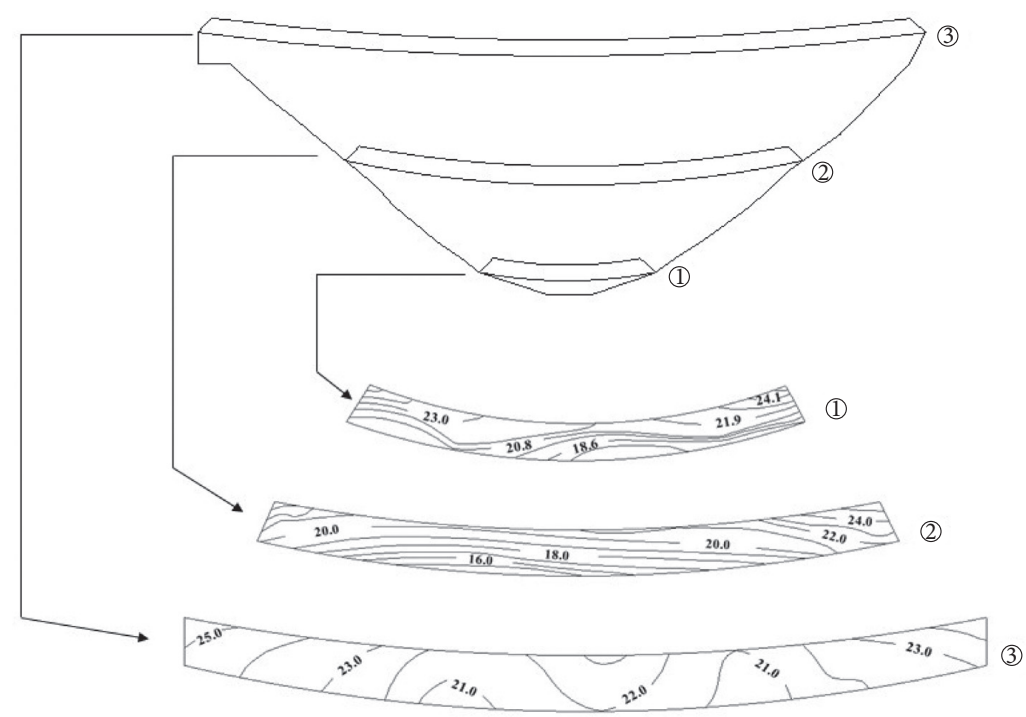

(b)

FIgURE 7: Temperature field diagrams of typical sections. (a) Vertical dam sections. (b) Horizontal dam sections.

Table 1: Average relative errors of the dam sections.

\begin{tabular}{lccr}
\hline Average relative error & No. & AdaBoost-ANN (\%) & Temperature extrapolation (\%) \\
\hline & $9 \#$ & 0.47 & 5.32 \\
Typical dam sections & $15 \#$ & 0.53 & 3.98 \\
& $22 \#$ & 0.30 & 4.91 \\
& $29 \#$ & 0.35 & 5.13 \\
& $35 \#$ & 0.59 & 5.02 \\
Atypical dam sections & $6 \#$ & 0.38 & 6.16 \\
& $12 \#$ & 0.47 & 5.21 \\
& $18 \#$ & 0.51 & 5.66 \\
& $24 \#$ & 0.45 & 5.92 \\
\end{tabular}


Therefore, the AdaBoost-ANN algorithm can accurately calculate the temperature field of the arch dam, its accuracy is higher than that of the temperature extrapolation method, and its computational cost is lower than that of FEM.

\section{Conclusions}

Temperature has an important impact on the establishment of the safety index and safety operation of concrete arch dams. Timely and accurately obtaining the temperature field of the concrete arch dam has important engineering significance. The reconstruction of the overall temperature field of the concrete arch dam based on the measured temperature values of several typical sections mainly includes the temperature extrapolation method and the finite element method. The temperature extrapolation method is easy to calculate, but the result is very different from the actual temperature field of the concrete arch dam. The result obtained by the finite element method is closer to the actual temperature field of the concrete arch dam, but each calculation needs to redetermine the boundary conditions, which is time-consuming and laborious. In this paper, the AdaBoost-ANN algorithm is used to reconstruct the temperature field of the concrete arch dam. The results are accurate, and the calculation process saves time and effort.

The AdaBoost-ANN algorithm has been widely used in engineering in recent years. This method avoids the overfitting phenomenon that ANN algorithm is prone to, improves the accuracy of the results, and has strong generalization performance. Taking the Xiaowan Arch Dam as an example, the temperature field model of the concrete arch dam is established by the AdaBoost-ANN algorithm. It can be seen from Figures 6 and 7 and Table 1 that the AdaBoost-ANN algorithm can accurately establish the temperature field of the concrete arch dam, and the results meet the accuracy requirements of reconstructing the temperature field of the arch dam.

In practical applications, the AdaBoost-ANN algorithm can be used not only for concrete arch dams to update the temperature field based on measured temperature values, but also for concrete gravity dams and other temperaturesensitive projects. As long as there are a certain number of real-time temperature measurement points, the AdaBoostANN algorithm can be used, and different weak classifications can be used to establish the temperature field model to obtain the real-time temperature field of the structure, and then the safety index of the structure can be determined. In addition, it should be noted that the AdaBoost-ANN algorithm is suitable for stable temperature conditions. For extreme temperature conditions, the AdaBoost-ANN algorithm is not suitable, and FEM is suitable for obtaining the temperature field. And if some measuring points are damaged, the temperature field established by the AdaBoostANN algorithm should be reconstructed.

\section{Data Availability}

All data generated or analyzed during this study are included within the article.

\section{Conflicts of Interest}

The authors declare that they have no conflicts of interest regarding the publication of this paper.

\section{References}

[1] C. O. Yigit, S. Alcay, and A. Ceylan, "Displacement response of a concrete arch dam to seasonal temperature fluctuations and reservoir level rise during the first filling period: evidence from geodetic data," Geomatic Natural Hazards \& Risk, vol. 7, no. 4, pp. 1-17, 2016.

[2] D. A. Arnold, "Thermal-optical design of a geodetic satellite for one millimeter accuracy," Advances in Space Research, vol. 65 , no. 10, pp. 2276-2289, 2020.

[3] G. A. Diego, G. L. Javier, and S. Jose, "A new approach for structural monitoring of large dams with a three-dimensional laser scanner," Sensors, vol. 8, no. 8, pp. 5866-5883, 2008.

[4] P. Breuer, T. Chmielewski, P. Górski, and E. Konopka, "Application of GPS technology to measurements of displacements of high-rise structures due to weak winds," Journal of Wind Engineering and Industrial Aerodynamics, vol. 90, no. 3, pp. 223-230, 2002.

[5] D. D. Maken, P. Leger, and S.-N. Roth, "Seasonal thermal cracking of concrete dams in northern regions," Journal of Performance of Constructed Facilities, vol. 28, no. 4, pp. 757-766, 2013.

[6] P. Léger and M. Leclerc, "Hydrostatic, temperature, timedisplacement model for concrete dams," Journal of Engineering Mechanics, vol. 133, no. 3, pp. 267-277, 2007.

[7] F. Sheibany and M. Ghaemian, "Effects of environmental action on thermal stress analysis of Karaj concrete arch dam," Journal of Engineering Mechanics, vol. 132, no. 5, pp. 532-544, 2006.

[8] F. Jin, Z. Chen, J. T. Wang, and J. Yang, "Practical procedure for predicting non-uniform temperature on the exposed face of arch dams," Applied Thermal Engineering, vol. 30, no. 1415, pp. 2146-2156, 2010.

[9] J. Mata, A. Tavares de Castro, and J. Sá da Costa, "Constructing statistical models for arch dam deformation," Structural Control and Health Monitoring, vol. 21, no. 3, pp. 423-437, 2014.

[10] J. Mata, "Interpretation of concrete dam behaviour with artificial neural network and multiple linear regression models," Engineering Structures, vol. 33, no. 3, pp. 903-910, 2011.

[11] J. Mata, A. Tavares de Castro, and J. Sá da Costa, "Timefrequency analysis for concrete dam safety control: correlation between the daily variation of structural response and air temperature," Engineering Structures, vol. 48, pp. 658-665, 2013.

[12] N. Shi, Y. Chen, and Z. Li, "Crack risk evaluation of early age concrete based on the distributed optical fiber temperature sensing," Advances in Materials Science and Engineering, vol. 2016, Article ID 4082926, 13 pages, 2016.

[13] Ministry of Water Resources of the People's Republic of China, SL 282-2018, Design Specification for Concrete Arch Dams, Ministry of Water Resources of the People's Republic of China, Beijing, China, 2018.

[14] J. G. Li, "An outward extension of FEM and its application to the calculation of temperature field," Design of Hydroelectric Power Station, vol. 9, no. 2, pp. 36-39, 1993.

[15] X.-F. Zhang, S.-Y. Li, Y.-L. Chen, and J.-R. Chai, "The development and verification of relocating mesh method for the 
computation of temperature field of RCC dam," Advances in Engineering Software, vol. 40, no. 11, pp. 1119-1123, 2009.

[16] V. Kuzmanovic, L. Savic, and J. Stefanakos, "Long-term thermal two- and three-dimensional analysis of roller compacted concrete dams supported by monitoring verification," Canadian Journal of Civil Engineering, vol. 37, no. 4, pp. 600-610, 2010.

[17] L. Li, X. Liu, V. T. N. Dao, and Y. Cheng, "Thermal cracking analysis during pipe cooling of mass concrete using particle flow code," Advances in Materials Science and Engineering, vol. 2016, Article ID 5976862, 10 pages, 2016.

[18] E. Castilho, N. Schclar, C. Tiago, and M. L. B. Farinha, "FEA model for the simulation of the hydration process and temperature evolution during the concreting of an arch dam," Engineering Structures, vol. 174, pp. 165-177, 2018.

[19] Y. Li, T. Bao, J. Gong, X. Shu, and K. Zhang, "The prediction of dam displacement time series using STL, extra-trees, and stacked LSTM neural network," IEEE Access, vol. 8, pp. 94440-94452, 2020.

[20] S.-K. Chen, Q.-D. He, and J.-G. Cao, "Seepage simulation of high concrete-faced rockfill dams based on generalized equivalent continuum model," Water Science and Engineering, vol. 11, no. 3, pp. 250-257, 2018.

[21] B. F. Zhu, Thermal Stresses and Temperature Control of Mass Concrete, Butterworth-Heinemann, London, UK, 2013.

[22] Y. Liu, G. X. Zhang, B. F. Zhu, and F. Shang, "Actual working performance assessment of super-high arch dams," Journal of Performance of Constructed Facilities, vol. 30, no. 2, 2016.

[23] M. Azmi and P. Paultre, "Three-dimensional analysis of concrete dams including contraction joint non-linearity," Engineering Structures, vol. 24, no. 6, pp. 757-771, 2002.

[24] J. Feng, H. Wei, J. Pan, Y. Jian, J. Wang, and C. Zhang, "Comparative study procedure for the safety evaluation of high arch dams," Computers and Geotechnics, vol. 38, no. 3, pp. 306-317, 2011.

[25] Q. Ren, L. Xu, and Y. Wan, "Research advance in safety analysis methods for high concrete dam," Science in China Series E: Technological Sciences, vol. 50, no. S1, pp. 62-78, 2007.

[26] P. Zvanut, G. Turk, and A. Kryzanowsk, "Effects of changing surrounding conditions on the thermal analysis of the moste concrete dam," Journal of Performance of Constructed Facilities, vol. 30, no. 3, 2016.

[27] Z. Wang, S. Liu, L. Vallejo, and L. Wang, "Numerical analysis of the causes of face slab cracks in Gongboxia rockfill dam," Engineering Geology, vol. 181, pp. 224-232, 2014.

[28] V.-R. Tarnawski and W. H. Leong, "Thermal conductivity of soils at very low moisture content and moderate temperatures," Transport in Porous Media, vol. 41, no. 2, pp. 137-147, 2000.

[29] D. Santillan, E. Saleteb, M. A. Toledob, and A. Granados, "An improved 1D-model for computing the thermal behaviour of concrete dams during operation. Comparison with other approaches," Computers and Concrete, vol. 15, no. 1, pp. 103-126, 2015.

[30] P. Léger, J. Venturelli, and S. S. Bhattacharjee, "Seasonal temperature and stress distributions in concrete gravity dams. Part 1: modelling," Canadian Journal of Civil Engineering, vol. 20, no. 6, pp. 999-1017, 1993.

[31] P. Léger, J. Venturelli, and S. S. Bhattacharjee, "Seasonal temperature and stress distributions in concrete gravity dams. Part 2: behaviour," Canadian Journal of Civil Engineering, vol. 20, no. 6, pp. 1018-1029, 1993.
[32] D. Santillán, E. Salete, D. J. Vicente, and M. A. Toledo, "Treatment of solar radiation by spatial and temporal discretization for modeling the thermal response of arch dams," Journal of Engineering Mechanics, vol. 140, no. 11, 2014.

[33] S. Qiang, Y. M. Zhu, S. Y. Wu, F. J. Chen, and Y. Zhang, "Temperature field and stress field simulation analysis for jinping-1 high arch dam," Water Resources and Power, vol. 2, pp. 50-52, 2007.

[34] X. P. Yang, X. L. Chang, and X. H. Liu, "FEM simulation of temperature and thermal stress of xiaowan arch dam," Applied Mechanics and Materials, vol. 212-213, pp. 887-890, 2012.

[35] Y. Freund and R. E. Schapire, "A decision-theoretic generalization of on-line learning and an application to boosting," Journal of Computer and System Sciences, vol. 55, no. 1, pp. 119-139, 1997.

[36] Y. Freund and R. E. Schapire, "A short introduction to boosting," Journal of Japanese Society for Artificial Intelligence, vol. 14, no. 5, pp. 771-780, 1999.

[37] Y. Cao, Q.-G. Miao, J.-C. Liu, and L. Gao, "Advance and prospects of AdaBoost algorithm," Acta Automatica Sinica, vol. 39, no. 6, pp. 745-758, 2013.

[38] C.-H. Loh, C.-H. Chen, and T.-Y. Hsu, "Application of advanced statistical methods for extracting long-term trends in static monitoring data from an arch dam," Structural Health Monitoring, vol. 10, no. 6, pp. 587-601, 2011.

[39] X. F. Li and G. Z. Liu, "The improvement of BP algorithm and its application," Journal of Sichuan University (Engineering Science Edition), vol. 32, no. 3, pp. 105-109, 2000.

[40] K. Ma, J. Liu, and H. S. Sun, "Application of the BP artificial neural network in dam's dispatching and management ability assessment," Computer Systems \& Applications, vol. 26, no. 12, pp. 250-256, 2017.

[41] P. Wang, L. Zhu, Q. Zhu et al., "An application of back propagation neural network for the steel stress detection based on Barkhausen noise theory," NDT \& E International, vol. 55, pp. 9-14, 2013.

[42] S. Haykin, Neural Networks and Learning Machines, Machine Press, Beijing, China, 2011.

[43] I. A. Basheer and M. Hajmeer, "Artificial neural networks: fundamentals, computing, design, and application," Journal of Microbiological Methods, vol. 43, no. 1, pp. 3-31, 2000.

[44] Z. Chen, D. Zheng, J. Shen, J. Qiu, and Y. Liu, "Research on distributed optical-fiber monitoring of biaxial-bending structural deformations," Measurement, vol. 140, pp. 462-471, 2019.

[45] H. Malik, M. K. Tarkeshwar, A. K. Yadav, and B. A. Krumar, "Application of physical-chemical data in estimation of dissolved gases in insulating mineral oil for power transformer incipient fault diagnosis with ANN," International Journal of Computer Applications in Technology, vol. 41, no. 21, pp. 43-50, 2012. 